\title{
Laboratory Experiments of Titan Tholins formed by Photochemistry of Cyanopolyynes
}

\author{
I. Couturier-Tamburelli ${ }^{1}$, I. N. Piétri ${ }^{1}$ \\ ${ }^{1}$ Aix Marseille Université, Laboratoire PIIM, UMR 6633, 13397 Marseille cedex 20 France.
}

Tholins are complex organic materials produced by irradiation of several carbon and nitrogen rich atmosphere. It has been proposed that Tholins could have played an important role in the origin of life on Earth [1]. We investigate the formation of polymer (Tholins) from the photolysis of dicyanoacetylene.

As of today, nitriles molecules have been identified in Titan atmosphere. Among these nitriles, the cyanopolyynes $\left(\mathrm{HC}_{\mathrm{n}} \mathrm{N}\right)$ are very important since they are the essential constituents in building block amino acids. It is known that a rich phochemistry takes place in the Titan aerosols, and contributes to the evolution of molecular diversity in this atmosphere. These compounds evolve through polymerization processes in aerosol particles, which grow by coagulation and rain down to the surface of Titan containing water ice.

We present photochemical processes of larger cyanopolyyne formation from small precursor molecules submitted to long wavelength photons. Under UV irradiation cyanopolyynes are known to induce izomerization process (figure 1) [2] and formation of longer cyanopolyynes [3].

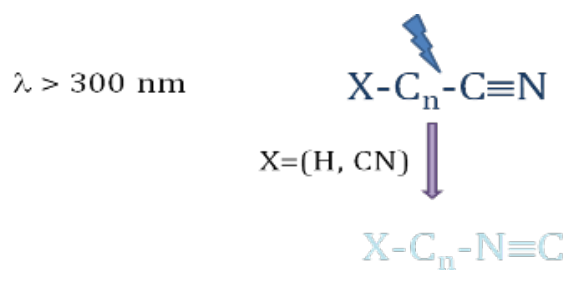

Figure 1: Izomerisation process of cyanopolyynes.

We provide the photochemical processes of Titan Tholins formation (figure 2) from cyanopolyyne precursor molecules submitted to long wavelength photons. Such photons penetrate down into the stratosphere and troposphere (figure 3). The photoreactivity of the cyanopolyynes with other Titan molecules are also presented. Laboratory experiments involving ultraviolet irradiation of dicyanoacetylene $\left(\mathrm{C}_{4} \mathrm{~N}_{2}\right)$ trapped in water ice have been conducted and monitored by infrared spectroscopy (FTIR). The irradiation of a $\mathrm{C}_{4} \mathrm{~N}_{2} / \mathrm{H}_{2} \mathrm{O}$ ice mixture at long wavelenghts has been found to be a possible source of $\mathrm{NH}_{4}{ }^{+} \mathrm{HCO}_{3}{ }^{-}$(ammonium bicarbonate) and $\mathrm{NH}_{4}{ }^{+} \mathrm{HCOO}^{-}$(ammonium formate) [4]. 


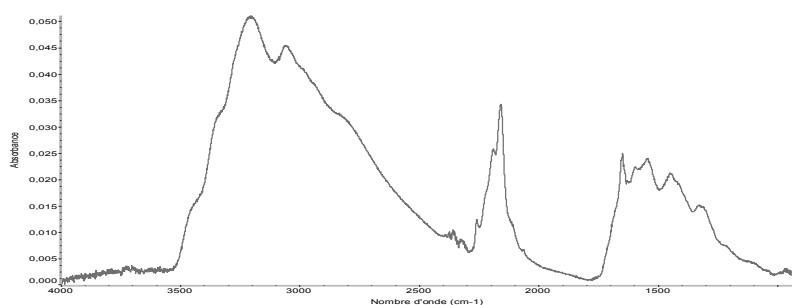

Figure 2: Infrared spectrum of Titan Tholins obtained by photolysis of pure $\mathrm{C}_{4} \mathrm{~N}_{2}$ ice.

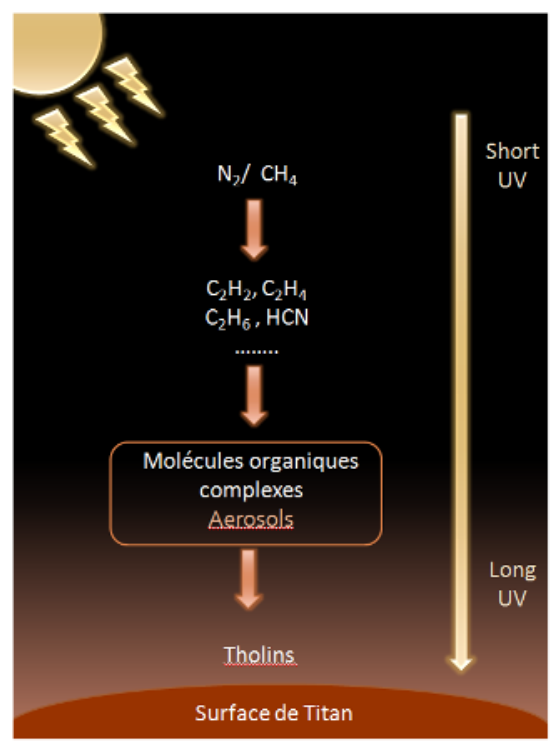

Figure 3: Titan atmosphere and surface.

[1] Sagan, C., Khare, B. 1979, Nature 277, 102-107.

[2] Coupeaud A, Pietri N, Couturier-Tamburelli I, Aycard JP, 2005, Chemical Physics Letters , 416, 4-6, 349.

[3] Coupeaud A, Kolos R, Couturier-Tamburelli I, Aycard JP, Pietri N, 2006, Journal Of Physical Chemistry A 110, 7, 2371.

[4] Guennoun Z, Pietri N, Couturier-Tamburelli I, Aycard JP, Journal Of Physical Chemistry A, 2006 110, 24, 77. 